\title{
Validity of the acoustic approximation for elastic waves in heterogeneous media.
}

\author{
Cance Philippe ${ }^{1}$, Capdeville Yann ${ }^{1}$ \\ 1 Laboratoire de Planétologie et Géodynamique de Nantes, CNRS, Université de Nantes \\ email : philippe.cance@univ-nantes.fr
}

\begin{abstract}
The acoustic approximation of elastic waves is a very common approximation in exploration geophysics. The interest of the acoustic approximation in the inverse problem context lies in the fact that it leads to a much lower numerical cost than for the elastic problem. Nevertheless, the Earth is not an acoustic body and it has been shown in the past that this approximation is not without drawbacks mainly because $\mathrm{P}$ to $\mathrm{S}$ energy conversion and anisotropy cannot be easily modelled. Here, we study a different issue of this approximation related to small size heterogeneities with respect to the minimum wavelength of the wavefield. We first numerically show that elastic and acoustic waves behave differently with respect to small scale heterogeneities, introducing not only differences in amplitudes but also in phase between elastic and acoustic signals. We give then physical and mathematical interpretations of this phenomenon, showing the different nature of elastic and acoustic wave propagation and leading to the conclusion that, in rough media, acoustic waves can only be a poor quality approximation of elastic waves. Interestingly, we also show that, in the acoustic case, small scale heterogeneities give rise to natural acoustic effective anisotropic media through an anisotropic effective mass matrix. Unfortunately, this anisotropy is of different nature compared to the elastic effective anisotropy and cannot be used to mimic elastic anisotropy.
\end{abstract}

\section{INTRODUCTION}

In the exploration geophysics context, the acoustic wave equation is often used as an approximation to the elastic wave equation. This approximation is widely used for migration techniques (e.g. Berkhout (1984); Etgen et al. (2009)), but also for full waveform inversion techniques (Virieux and Operto, 2009) for both theoretical developments (e.g. Tarantola (1984); Gauthier et al. (1986); Bunks et al. (1995); Pratt et al. (1998); Ben-Hadj-Ali et al. (2008); Métivier et al. (2013)) and data applications (e.g. Shin and Cha (2008); Plessix et al. (2010); Vigh et al. (2010); Schiemenz and Igel (2013)). The main reason to perform such an approximation is to simplify both forward and inverse problems. For the forward modeling, the acoustic approximation considerably reduces the numerical cost for two main reasons. Firstly, acoustic equations are scalar equations while elastic equations rely on vector and tensor quantities. Secondly, acoustic forward modeling may gain up to a factor 100 in computation time as the space sampling (and then time sampling) of the minimum wavelength of the wavefield relies on $\mathrm{P}$-wave velocities in the acoustic case while it relies on the much smaller S-wave velocities in the elastic case. For the full waveform inverse problem, a faster forward problem is a decisive advantage, and having to invert for fewer parameters than for the elastic case leads to a simpler inverse problem. If the reasons that motivate such an approximation are clear, its justifications are not that obvious. Indeed, the Earth is not an acoustic body and, even for marine exploration setup for which both sources and receivers lie in an acoustic medium, waves under consideration are elastic waves for most of the propagation path between the source and the receiver. In the migration context, where it is attempted to migrate only early $\mathrm{P}$ arrivals, this approximation seems often justified, at least 
when no anisotropy is present. In the full waveform inversion context however, this approximation doesn't seem always justified as it leads to persistent unreliable inversion results when $\mathrm{S}$-wave parameters play a non negligible role in P-wave propagation (Barnes and Charara, 2008). In the forward modeling context, acoustic approximations of elastic P-waves is well known for encountering major difficulties (Alkhalifah, 1998, 2000, 2003; Grechka et al., 2004; Fletcher et al., 2008; Operto et al., 2009; Bakker and Duveneck, 2011; Chu et al., 2012; Bube et al., 2012; Wu and Alkhalifah, 2014). Indeed, if the acoustic approximation of elastic $\mathrm{P}$-waves is natural for homogeneous isotropic media, it is not the case anymore for anisotropic media nor for heterogeneous media where P-to-S conversions may occur but cannot be taken into account by acoustic equations (although one can find a method for correcting this issue in some cases in Hobro et al. (2014)). As both scattering and effective anisotropy are observed on elastic wavefields propagating with wavelengths much larger than the size of the medium's heterogeneities, we focus here on the accuracy of acoustic approximations for elastic $\mathrm{P}$-waves in 'rough' heterogeneous media, that is when heterogeneity scales smaller than the minimum wavelength are present. To address this problem, we first recall the elastic and acoustic wave equations and how they can be related to each other in the homogeneous media case. We present then three simple numerical experiments to challenge the acoustic approximation of elastic wave propagation. After showing that the approximation fails for "rough" media we mathematically and physically propose an explanation for this disagreement based on homogenization theory. Finally we discuss the induced effective acoustic anisotropy versus elastic anisotropy in the simple layered case before concluding.

\section{ACOUSTIC APPROXIMATION OF THE ELASTIC EQUATIONS}

Considering an elastic domain $\boldsymbol{\Omega}$ with a free surface $\partial \boldsymbol{\Omega}$, the elastic wave equations driving the displacement vector $\mathbf{u}(\mathbf{x}, t)$ are :

$$
\begin{aligned}
& \rho \ddot{\mathbf{u}}-\nabla \cdot \boldsymbol{\sigma}=\mathbf{f} \text { in } \boldsymbol{\Omega} \\
& \boldsymbol{\sigma}=\mathbf{c}: \boldsymbol{\epsilon}(\mathbf{u}) \text { in } \boldsymbol{\Omega} \\
& \boldsymbol{\sigma} \cdot \boldsymbol{n}=0 \text { in } \partial \boldsymbol{\Omega}
\end{aligned}
$$

where $\mathbf{x}$ is the positional vector, $t$ is the time component, $\mathbf{c}(\mathbf{x})$ is the elastic stiffness tensor, $\rho(\mathbf{x})$ is the (mass) density, $\boldsymbol{\sigma}$ is the stress tensor, $\boldsymbol{\epsilon}=\left(\nabla \mathbf{u}+{ }^{T} \nabla \mathbf{u}\right) / 2$ is the strain tensor, ${ }^{T}$ denotes the transpose operator, $\mathbf{f}$ is the source term and $\boldsymbol{n}$ denotes the unit normal to $\partial \boldsymbol{\Omega}$. In general, the elastic stiffness tensor $\mathbf{c}$ depends upon 21 independent coefficients in 3-D and 6 in 2-D, but only on the two Lamé parameters $\lambda(\mathbf{x})$ and $\mu(\mathbf{x})$ for the isotropic case (Lamé, 1852). In such a case, the constitutive relation (2) can be rewritten as :

$$
\boldsymbol{\sigma}=\lambda \operatorname{tr}(\boldsymbol{\epsilon}) \mathbf{I}+2 \mu \boldsymbol{\epsilon}
$$

where $\mathbf{I}$ is the identity tensor and $\operatorname{tr}(\mathbf{A})=\mathbf{A}_{i i}$ (using the Einstein implicit summation convention) is the trace operator. Isotropic P-wave and S-wave velocities are then related to Lamé parameters through :

$$
\begin{aligned}
& \lambda+2 \mu=\rho V_{P}{ }^{2} \\
& \mu=\rho V_{S}{ }^{2}
\end{aligned}
$$

For the same domain $\boldsymbol{\Omega}$, but for the acoustic wave propagation, the velocity potential $q$ is solution of :

$$
\begin{aligned}
& \frac{1}{\kappa} \ddot{q}-\nabla \cdot \dot{\mathbf{u}}=\dot{g} \text { in } \boldsymbol{\Omega} \\
& \dot{\mathbf{u}}=\frac{1}{\rho} \nabla q \text { in } \boldsymbol{\Omega} \\
& q=0 \text { in } \partial \boldsymbol{\Omega}
\end{aligned}
$$

where $\kappa(\mathbf{x})$ is the acoustic bulk modulus, $\rho(\mathbf{x})$ is the (mass) density, $\mathbf{u}(\mathbf{x}, t)$ is the displacement vector and $g(\mathbf{x}, t)$ is a scalar source term. In general, the acoustic medium is fully described by only 2 parameters, namely its density $\rho(\mathbf{x})$ and the 'sound speed' $V(\mathbf{x})$ such that $\kappa(\mathbf{x})=\rho(\mathbf{x}) V^{2}(\mathbf{x})$, where the acoustic bulk modulus $\kappa$ shall not be confused with the elastic bulk modulus $K=$ $\lambda+\frac{2}{3} \mu$ (which is not used in this paper).

In general, the displacement vector $\mathbf{u}$ solution to the above elastodynamic equations can be related to its acoustic counterpart only for an infinite homogeneous isotropic domain and for an explosive isotropic source as described in Appendix A. However, realistic seismic wave propagation in the Earth necessitates at least a free surface and some reflectors. Reflections will then occur at each interface generating $S$ waves, which breaks the assumption that the displacement $\mathbf{u}$ can derive only from the above potential $q$. A solution to this problem is to "filter" rotational waves ( $\mathrm{S}$ waves), keeping only the irrotational part of the signal which ought to derive from the wanted potential. To do so, we use the pressure wavefield $p$ defined as $p=-(1 / 2) \kappa \nabla \cdot \mathbf{u}$ in the elastic case (Landau and Lifshitz, 1959b). Indeed, no rotational part of the original displacement $\mathbf{u}$ is left in $p$ since $\nabla \cdot(\nabla \times \mathbf{m})=0$ for any vector $\mathbf{m}$. We compare then the elastic pressure $p$ to the acoustic pressure signal defined as $\dot{q}$ (Landau and Lifshitz, 1959a). In practice, P-to-S (and S-to-P) conversions cannot be accounted for by acoustic approximations anyway, leading to incorrect modeling of amplitudes for elastic Pwaves. Phases however may still be correctly modeled in isotropic homogeneous media (with reflections), at least for the first arrivals. In the next two sections, we numerically investigate the equivalence of acoustic and elastic waves in non-homogeneous media with respect to the pressure signal's phase.

\section{Heterogeneous media : the smooth and rough cases}

As shown in the previous section, the acoustic and elastic wave equation solution equivalence for P-waves only holds 
theoretically in the case of a constant infinite medium. Nevertheless, it is reasonable to assume that the approximation is still valid in the quasi homogeneous (very smooth) media case. In practice, this approximation is used in heterogeneous media that most of the time contains small scale heterogeneities, media to which we will refer as rough media. In the next section, we numerically investigate the validity and the behavior of the acoustic approximation of elastic waves in both smooth and rough media.

Before moving further, we define more precisely "smooth" and "rough" media. To do so, we first define the medium roughness scale $\lambda_{0}{ }^{*}$. $\lambda_{0}$ is a characteristic measurement of the spatial variations of elastic and acoustic medium parameters. For example, it can be related to the minimum thickness of the layers in a discontinuous layered medium or to the smallest oscillation scale of a continuous medium. Second, we need an estimate of the minimum wavelength of the wavefield $\lambda_{\text {min }}$. Knowing the maximum frequency $f_{c}$ (corner frequency of the source or maximum frequency of the filtered seismograms) and the minimum wave velocity over the whole domain $V_{\text {min }}$, a lower bound estimate of $\lambda_{\min }$ is $V_{\min } / f_{c}$. In general, $S$ waves being always slower than $P$ waves, $V_{\min }$ should be set using the slowest $S$ waves. However, we are primarily interested in $P$ waves and we will use the minimum $P$ wave velocity to estimate $V_{\text {min }}$. Defining the parameter $\varepsilon_{0}=\lambda_{0} / \lambda_{\text {min }}$ to describe the roughness of the medium toward the wavefield, the following two cases will be considered :

- $\varepsilon_{0} \gg 1$, the smooth medium case;

- $\varepsilon_{0} \ll 1$, the rough medium case.

\section{Numerical experiments of the acoustic ap- proximation for heterogeneous smooth and rough media}

To study the effect of heterogeneity scale on the acoustic approximation of elastic P-waves, we use three different 2$\mathrm{D}$ experiments and for each experiment type, both smooth and rough models are introduced :

1. experiment (1): periodic continuous stratified media (Figure 1);

2. experiment (2): 2-D random media (Figure 2);

3. experiment (3): 2-D SEAM ${ }^{\dagger}$-based media (Figure 3).

For each medium, we first define the elastic version by defining the density $\rho(\mathbf{x})$, and either both Lamé parameters $\lambda(\mathbf{x})$ and $\mu(\mathbf{x})$ or both $\mathrm{P}$-wave and S-wave velocities $V_{P}(\mathbf{x})$ and $V_{S}(\mathbf{x})$ respectively over the physical domain $\boldsymbol{\Omega}$. Then the acoustic model version is defined as for the homogeneous case by using density and $\mathrm{P}$-waves velocities from the elastic case : $V=V_{P}$ (and we therefore always have $\kappa=\lambda+2 \mu=\mathbf{c}_{1111}=\mathbf{c}_{2222}$ ).

\footnotetext{
${ }^{*}$ Contrary to the Lamé parameter $\lambda$, wavelengths or scales such as $\lambda_{0}$ will always be subscripted.

${ }^{\dagger}$ Fehler and Larner (2008)
}

Experiment (1) media

For the experiment (1), the layered medium is continuously defined vertically ( $x_{2}$ component) as a sine function around an average value for the density $\rho$ and the Lamé parameters (see Figure 1) :

$$
\begin{aligned}
& \rho\left(x_{2}\right)=\langle\rho\rangle\left(1+\nu_{\rho} \cos \frac{2 \pi x_{2}}{\lambda_{0}}\right) \\
& \lambda\left(x_{2}\right)=\langle\lambda\rangle\left(1+\nu_{\lambda} \cos \frac{2 \pi x_{2}}{\lambda_{0}}\right) \\
& \mu\left(x_{2}\right)=\langle\mu\rangle\left(1+\nu_{\mu} \cos \frac{2 \pi x_{2}}{\lambda_{0}}\right)
\end{aligned}
$$

where $\lambda_{0}$ is the period, $\langle\rho\rangle,\langle\lambda\rangle$ and $\langle\mu\rangle$ are the average quantities, and $\nu_{\rho}=20 \%$ and $\nu_{\lambda}=\nu_{\mu}=55 \%$ are the contrasts on density and elastic parameters respectively. These contrasts may not reflect realistic media values but are chosen to make our point clear, as it will appear later. Knowing that using a source corner frequency $f_{c}=10 \mathrm{~Hz}$ leads to $\lambda_{P_{\text {min }}}=300 \mathrm{~m}$ and $\lambda_{S_{\text {min }}}=200 \mathrm{~m}$, we choose $\lambda_{0}=8.5 \mathrm{~km}$ for the smooth medium $\left(\varepsilon_{0} \sim 28\right)$ and $\lambda_{0}=$ $50 \mathrm{~m}$ for the rough medium $\left(\varepsilon_{0} \sim 0.17\right)$.
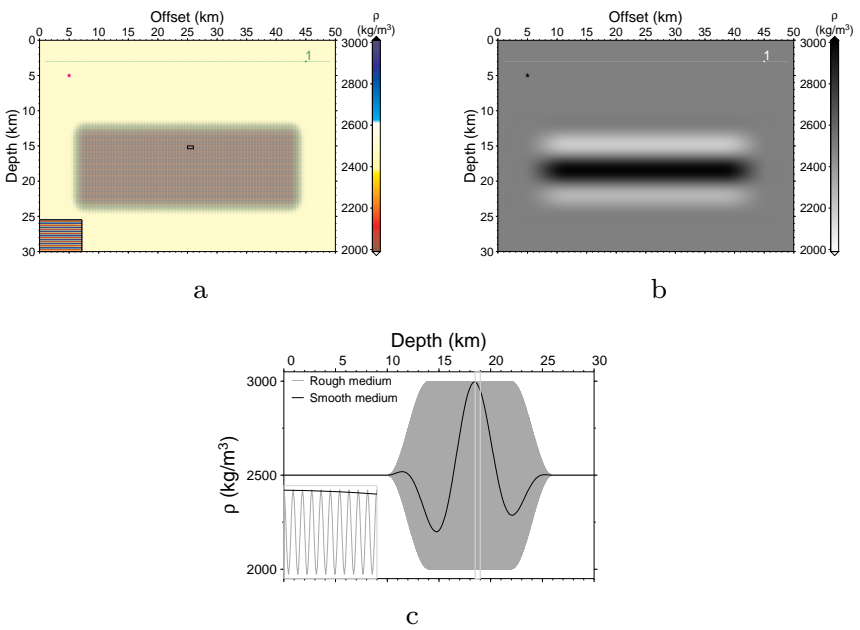

Figure 1: Stratified media for experiment (1). 1a and 1b: density maps of the rough and smooth medium respectively. In the rough case, we saturated image contrasts for visibility and lower left box is a zoom on the small scale varying medium corresponding to the $1 \mathrm{~km} \times 400 \mathrm{~m}$ central black box. The black star indicates the source location and white diamonds (white line) the receiver locations. 1c : vertical cross section for $x=15 \mathrm{~km}$ obtained for $\lambda_{0}=50 \mathrm{~m}$ (rough model, grey line) and $\lambda_{0}=8.5 \mathrm{~km}$ (smooth model, black line) with a zoom (lower left box corresponds to depths between $18.5 \mathrm{~km}$ and $19 \mathrm{~km}$ ).

\section{Experiment (2) media}

For the experiment (2), we first define the rough medium. It is defined as a matrix of $800 \times 900$ square elements of $20 \times 20 \mathrm{~m}^{2}$ with constant elastic properties. For each element, the density, $\mathrm{P}$-wave and $\mathrm{S}$-wave velocities are ran- 
domly chosen within a contrast $\nu=12.5 \%$ of their average value (we can then consider that Lamé coefficients - or elastic coefficients - are roughly taken within a contrast $\nu_{\mathbf{c}} \simeq 35 \%$ of their average value). For a source corner frequency $f_{c}=10 \mathrm{~Hz}$, we roughly have $\lambda_{P_{\text {min }}} \simeq 200 \mathrm{~m}$ $\left(\varepsilon_{0}=0.1\right)$ and $\lambda_{S_{\text {min }}} \simeq 100 \mathrm{~m}$ which is indeed large compared to $\lambda_{0}=20 \mathrm{~m}$. The smooth model is obtained by spatially low-pass filtering the rough model's density and velocities. To do so, we introduce (in Appendix B) a low pass filter operator $\mathcal{F}_{\lambda_{c}}()$ such that, for any spatially varying quantity $A(\mathbf{x}), \mathcal{F}_{\lambda_{c}}(A)(\mathbf{x})$ doesn't contain spatial variations smaller than $\lambda_{c}$. We use $\lambda_{0}=\lambda_{c}=1200 \mathrm{~m}$ to define the smooth model $\left(\varepsilon_{0}=6\right)$.

\section{Experiment (3) media}

For the experiment (3), we use a 2 -D cross section of the SEG's SEAM model (Fehler and Larner, 2008), defined on a $10 m \times 10 m$ regular grid. The rough model is obtained by applying a taper toward constant properties to the sides of the model and S-wave velocities under $1 \mathrm{~km} / \mathrm{s}$ have been clipped (implying that the top water layer has been replaced by an elastic medium). Small scale heterogeneities (with a pseudo-period of $\lambda_{0} \simeq 70 \mathrm{~m}$ ) and strong contrasts $\nu$ on density, P-waves velocity (Figure 3c) and S-wave velocity can be found on top $(\nu \simeq 5 \%)$ and under $(\nu \simeq 8 \%)$ the center salt structure. For a source corner frequency of $f_{c}=4 \mathrm{~Hz}$ the estimated P-wave minimum wavelength is $\lambda_{P_{\text {min }}} \simeq 375 \mathrm{~m}$ which can be considered as much larger than the medium roughness scale $\lambda_{0} \simeq 70 \mathrm{~m}$ $\left(\varepsilon_{0} \sim 0.2\right)$. Similarly to the experiment $(2)$, the smooth model is obtained from the rough model by applying the low-pass filter to the rough density and wave velocities with $\lambda_{0}=2500 \mathrm{~m}$. Using the previous source corner frequency $f_{c}=4 \mathrm{~Hz}$ gives then an estimated $\mathrm{P}$-wave minimum wavelength of $\lambda_{P_{\text {min }}} \simeq 280 \mathrm{~m}$ which is indeed much smaller than $\lambda_{0}$. However, despite the important ratio $\varepsilon_{0}=\lambda_{0} / \lambda_{P_{\min }} \simeq 9$, important large-scale contrasts on velocities over the domain do not allow for $\lambda_{P_{\text {min }}}$ to be a representative length to ensure that the filtered medium is really smooth. Indeed, the medium has to be smooth with respect to the main wavelength of the wavefield which is more accurately described by the central frequency of the source $\left(f_{0} \simeq f_{c} / 2.5\right)$ and for P-wave velocities encountered in the most part of the wave path. We estimate this main P-wave velocity at minimum as $V_{P} \simeq 2500 \mathrm{~m} / \mathrm{s}$, giving then a main wavelength of $V_{P} / f_{0} \simeq 1600 \mathrm{~m}$ which is not small enough compared to $\lambda_{0}$. We cannot smooth the medium any further (a correct cut-off frequency used to obtain a really smooth medium would then correspond to a wavelength of the order of the medium's depth, thus rendering the smooth medium almost homogeneous) and the only option left to define a smooth medium using the more reasonable medium roughness scale of $\lambda_{0}=2500 \mathrm{~m}$ is to increase the source corner frequency to $f_{c}=30 \mathrm{~Hz}$, allowing an estimated main wavelength for the wavefield of about $210 m \ll \lambda_{0}\left(\varepsilon_{0} \sim 12\right)$.

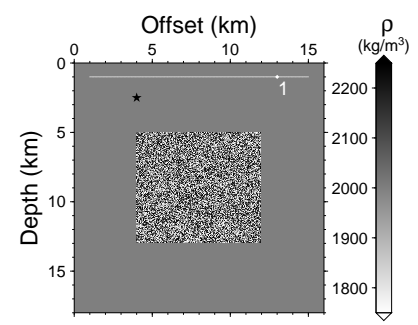

a

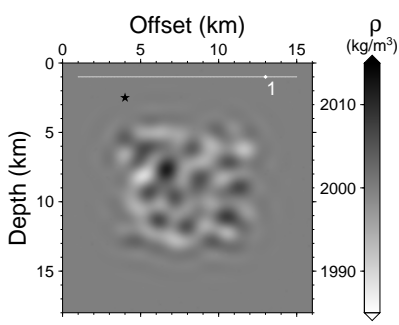

b

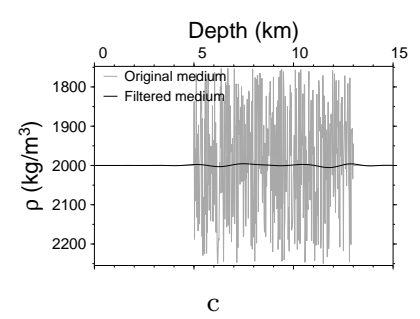

Figure 2: Random media for experiment (2). 2a and 2b : density maps of the rough and smooth medium respectively. The rough model contains square elements with constant elastic properties of size $\lambda_{0}=20 \mathrm{~m}$. The smooth model is a low-pass filtered version of the rough model with cut-off spatial frequency of $\lambda_{0}=1200 \mathrm{~m}$. The black star indicates the source location and white diamonds (white line) the receiver locations. $2 \mathrm{c}$ : vertical cross section for $x=5 \mathrm{~km}$ obtained for $\lambda_{0}=20 \mathrm{~m}$ (rough model, grey line) and $\lambda_{0}=1200 \mathrm{~m}$ (smooth model, black line).

\section{Experiments and numerical set up}

Each experiment is devised as a seismic reflection survey. For each experiment the source and receivers are located near the top surface. In order to simplify the wavefield analysis, the top boundary is treated with an absorbing boundary condition instead of a free surface boundary condition, thus eliminating the free surface reflected down-going wave. Lateral domain boundaries are also defined as absorbing boundaries. The bottom boundary is set to a free surface condition which is unusual but remains a simple way to generate an upcoming reflected wave. We observe then a simple direct $\mathrm{P}$-wave (traveling only through a homogeneous medium) and waves coming back to the surface after traveling through the heterogeneous medium. To numerically solve the elastic and acoustic wave equations we use the Spectral Element Method (Komatitsch and Vilotte, 1998; Komatitsch and Tromp, 2002). The absorbing conditions are achieved using Perfectly Matched Layers (PMLs) (Festa and Vilotte (2005)'s version). For each experiment, the same mesh, spectral element polynomial basis and time sampling are used in both elastic and acoustic cases. We systematically mesh the discontinuities of the medium, if any, and elements size and number of $1 D$ Gauss-Lobatto-Legendre (GLL) points, see Table 1 , are always chosen to oversample the wavefield. This allows to obtain excellent numerical accuracy (see Appendix C) so that the observed differences between signals cannot be related to numerical artifacts. 


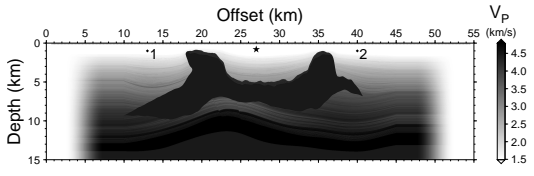

a
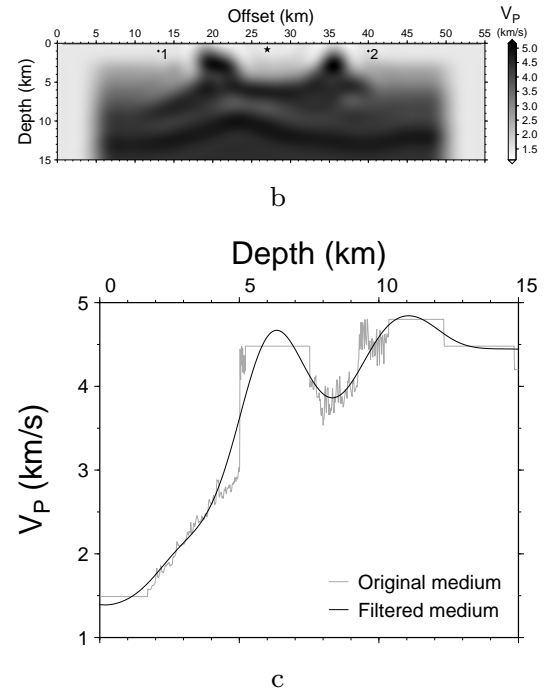

Figure 3: Adapted 2D cut in SEG's SEAM (3a,3b : Pwave velocity mappings, $3 \mathrm{c}$ : vertical $1 D$ cut at offset $x=27 \mathrm{~km})$. Original model (3a) is linearly interpolated from its original $10 m \times 10 m$ regular grid. Small scale heterogeneities with strongest contrasts are found on top of the salt structure with pseudo-period of $\lambda_{0} \simeq 70 \mathrm{~m}$. (3b) is a low-pass filtered version of (3a) with cut-off spatial frequency of $\lambda_{0}=2500 \mathrm{~m}$. Black star locates the source and black diamonds (including the top black line) locate receivers.

\begin{tabular}{|l|c|c|c|}
\hline Experiment & Element size $\left(\mathrm{m}^{2}\right)$ & GLL points & $\lambda_{P_{\min }}(\mathrm{m})$ \\
\hline 1 (rough) & $200 \times 25$ & 9 & 300 \\
\hline 1 (smooth) & $200 \times 200$ & 9 & 300 \\
\hline 2 (rough) & $20 \times 20$ & 5 & 200 \\
\hline 2 (smooth) & $20 \times 20$ & 5 & 200 \\
\hline 3 (rough) & $10 \times 10$ & 5 & 375 \\
\hline 3 (smooth) & $50 \times 50$ & 9 & 40 \\
\hline
\end{tabular}

Table 1: Element sizes and number of GLL points in each spatial direction for each experiment in either the rough and smooth cases. As an example, in a homogeneous medium and for 9 GLL points per edge of an element, SEM keeps a very good accuracy with up to $2 \lambda_{\min }$ per element.

To assess the accuracy of the acoustic approximation of elastic waves, we compare the acoustic pressure $\dot{q}$ to the elastic pressure $-(1 / 2) \kappa \nabla \cdot \mathbf{u}$, but not directly. Indeed, due to energy losses during P-to-S conversions for elastic wave propagation, the amplitude of the acoustic pressure is not expected to match the amplitude of the elastic pressure, and only the phase of the signal may be accurately modeled. For experiment (1) and (2), the signals are very simple making it possible to use a simple and crude way to perform phase comparisons : each wave arrival is normalized to the same amplitude in a given time window. Doing so, observed signal differences are only related to phase error.

\section{Smooth media results}

For the smooth case, results for experiments (1), (2) and (3) are respectively gathered in the left column of Figures 4, 5, and 6. For experiments (1) and (2), traces show mainly two well-separated arrivals - first the direct wave and then the wave reflected at the domain bottom - which are then individually normalized. For experiment (3) only one arrival can clearly be identified (Figure 6e) for almost every offsets. Consequently, the normalization is applied on the whole signal and not by arrival time windows as it should. The agreement between acoustic and elastic solutions for experiments (1) and (2) is excellent and the relative error $\left|\left(p_{e l}-p_{a c}\right) / p_{e l}\right|$ between the normalized elastic pressure $p_{e l}=-(1 / 2) \kappa \nabla \cdot \mathbf{u} / \max (-(1 / 2) \kappa \nabla \cdot \mathbf{u})$ and its (also normalized) acoustic counterpart $p_{a c}=\dot{q} / \max (\dot{q})$ is less than 1\% (Figures 4e and 5e). For experiment (3), the agreement between the two solutions is also very good (Figure 6e) except for some late arrivals in a small area. These late differences are due to $\mathrm{P}$ to $\mathrm{S}$ energy losses in the high velocity structure area (salt area).

For smooth models, it can be concluded that, as expected, the acoustic approximation of elastic waves is accurate, at least for time arrivals. 


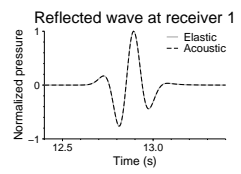

a

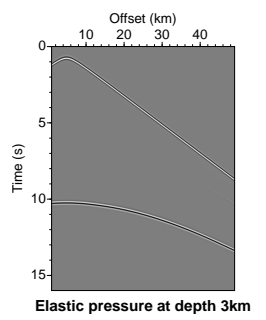

c

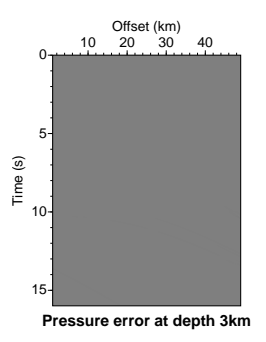

$\mathrm{e}$

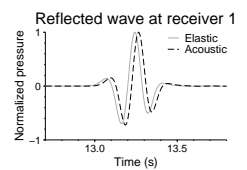

b

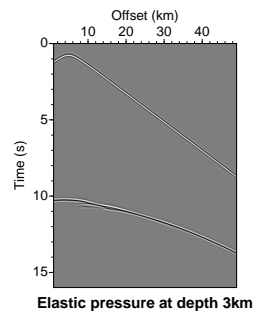

d

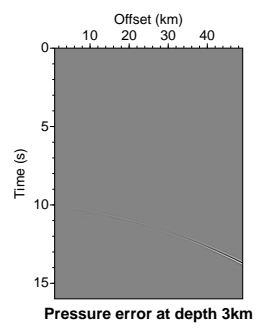

f

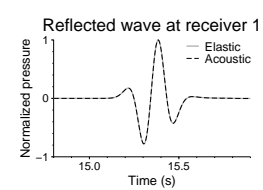

a

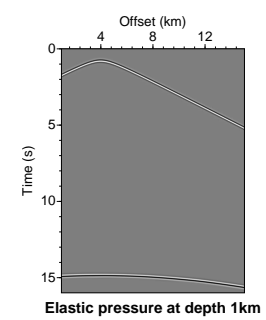

c

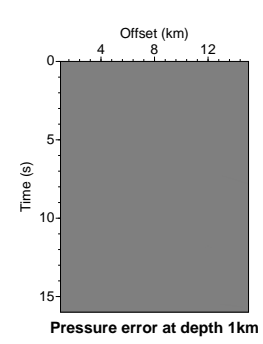

e

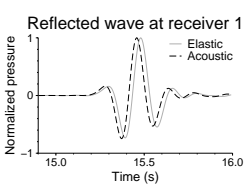

b

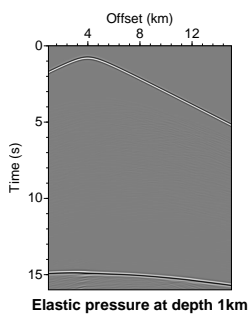

d

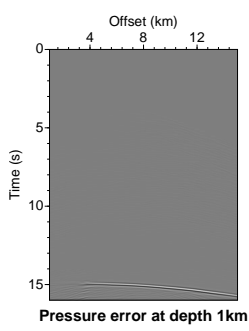

$f$
Figure 4: Experiment (1) : Stratified model - Left : smooth medium, right : rough medium ; $(4 \mathrm{a}$ and $4 \mathrm{~b})$ : elastic (grey) and acoustic (black) normalized pressure seismograms at receiver 1 ; $(4 \mathrm{c}$ and $4 \mathrm{~d})$ : normalized seismogram gathers for the elastic run; (4e and $4 \mathrm{f})$ : gathers of the error between elastic and acoustic normalized pressure seismograms.

\section{Rough media results}

For the rough case, results for experiments (1), (2) and (3) are respectively gathered in the right column of Figures 4,5 and 6 . For experiments (1) and (2), the acoustic approximation is accurate for the direct wave arrival, which is expected as the wave propagation path is homogeneous (Figures 4f, 5f). For the bottom reflected arrival, a significant phase shift is observed for both experiments (1) and (2). This phase shift is larger for increasing offsets for experiment (1) (Figure 4f) whereas it is almost offset independent for experiment (2) (Figure 5f). For experiment (3), observations are complicated by the fact that the amplitude normalization can not be performed efficiently, but a similar phase shift as for experiment (1) and (2), even though smaller, can be observed (Figures $6 \mathrm{~b}$ and $6 \mathrm{~d}$ ).

From these numerical observations we conclude that something more than just P-to-S energy conversion losses occur during elastic wave propagation in rough media : ballistic acoustic and elastic waves experience an overall different wave speed even though local wave speed are by construction the same. This is an indication that the physics of acoustic and elastic wave propagation are different. To understand this observation better physically, we propose to rely on the two scale homogenization the-
Figure 5: Experiment (2) : Random model - Left : smooth medium, right : rough medium ; (5a and $5 \mathrm{~b})$ : elastic (grey) and acoustic (black) normalized pressure seismograms at receiver $1 ;(5 \mathrm{c}$ and $5 \mathrm{~d})$ : normalized seismogram gathers for the elastic run ; (5e and $5 \mathrm{f})$ : gathers of the error between elastic and acoustic normalized pressure seismograms.

ory for both elastic waves (Backus, 1962; Capdeville et al., 2010a,b) and acoustic waves (treated as a special case of Guillot et al. (2010)). This is the purpose of the next section.

\section{HOMOGENIZATION FOR THE WAVE PROPAGATION PROBLEM}

In general, the two-scale homogenization method allows to compute large-scale-only 'effective' parameters and equations equivalent to an initial problem with both large and fine scales. It allows first, to better understand physical phenomena where small scales have only an effective role and second, to compute solutions of the initial problem at the effective scale only, thus generally significantly reducing the computing time and/or the meshing problem. Two-scale homogenization techniques have been studied for a long time for various problems in mechanics for periodic or non-periodic media (Auriault and SanchezPalencia, 1977; Bensoussan et al., 1978; Sanchez-Palencia, 1980; Allaire, 1992).

For wave propagation problems, two scale homogenization allows to understand how small scale heterogeneities are upscaled - or "seen" - by a long wavelength wavefield and therefore gives the opportunity to explain and 
interpret observations when the ratio heterogeneity scale versus minimum wavelength is very small compared to 1 : $\varepsilon_{0}=\lambda_{0} / \lambda_{\min } \ll 1$. We first briefly introduce the homogenization procedures for both elastic and acoustic waves in general heterogeneous media. Then, we detail the specific case of layered media - especially the periodic case - as they present some further theoretical results allowing for easier interpretations. Indeed, Backus (1962) showed on the particular case of layered media that the effective elastic stiffness tensor $\mathbf{c}^{*}$ may carry some effective anisotropy (also called 'extrinsic' anisotropy) computed through a non linear process recalled in the next section.

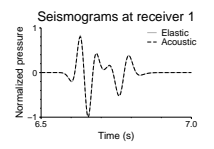

a

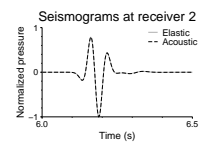

$\mathrm{c}$

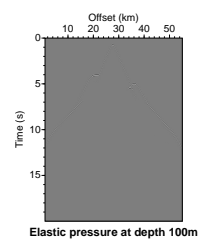

e

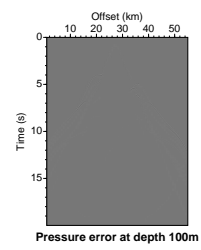

$\mathrm{g}$

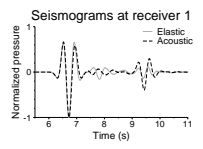

$\mathrm{b}$

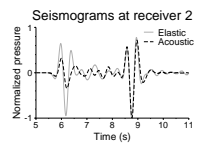

d
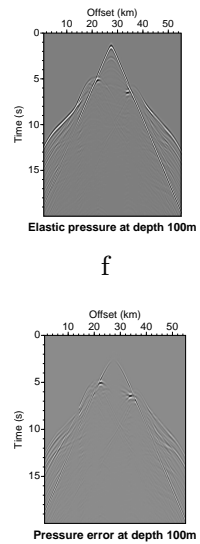

$\mathrm{h}$
Figure 6: Experiment (3) : SEAM-based model - Left : smooth medium, right : rough medium ; $(6 \mathrm{a}, 6 \mathrm{~b})$, and (6c, 6d) : elastic (grey) and acoustic (black) normalized pressure seismograms for receivers 1 and 2 respectively ; (6e and 6f) : seismogram gathers for the elastic run for reference ; $(6 \mathrm{~g}$ and $6 \mathrm{~h})$ : seismogram gathers for the error between elastic and acoustic runs.

\section{General 2-scale homogenization}

For the wave propagation problem in general heterogeneous non-periodic media, we will use deterministic homogenization procedures as defined in Capdeville et al. (2010b) for the elastic equations and as a special case of Guillot et al. (2010) for the acoustic equations.

For the elastic wave propagation case, assuming the density and elastic properties contain small scale variations with respect to $\lambda_{\text {min }}$, the order 0 homogenization approximates original equations $(1,2)$ with :

$$
\begin{aligned}
& \rho^{*} \ddot{\mathbf{u}}^{*}-\nabla \cdot \boldsymbol{\sigma}^{*}=\mathbf{f} \\
& \boldsymbol{\sigma}^{*}=\mathbf{c}^{*}: \boldsymbol{\epsilon}\left(\mathbf{u}^{*}\right)
\end{aligned}
$$

where $\rho^{*}$ is the effective density, $\mathbf{c}^{*}$ is the effective order4 elastic stiffness tensor. The effective displacement and stress $\left(\mathbf{u}^{*}, \sigma^{*}\right)$ approximate the 'real' displacement and stress. Using again a spatial low-pass filter $\mathcal{F}_{\lambda_{0}}$ (.) with a scale cut-off $\lambda_{0}$ (that is the action of $\mathcal{F}_{\lambda_{0}}$ (.) removes all variations smaller than $\lambda_{0}$, see Appendix B), the effective density is simply obtained as : $\rho^{*}=\mathcal{F}_{\lambda_{0}}(\rho)$. The effective elastic stiffness tensor $\mathbf{c}^{*}$ can not be obtained by only filtering the initial elastic stiffness tensor $\mathbf{c}$. General deterministic non periodic homogenization (Capdeville et al., 2010a,b) practically computes the effective elastic stiffness tensor through an order-3 tensor $\chi$ - called first order corrector - which is solution to the so called 'starting cell problem' :

$$
\begin{aligned}
& \nabla \cdot(\mathbf{H})=0 \\
& \mathbf{H}=\mathbf{c}: \mathbf{G} \\
& \mathbf{G}=\mathbf{I}+\frac{1}{2}\left(\nabla \chi+{ }^{T} \nabla \chi\right) \\
& \langle\chi\rangle=0
\end{aligned}
$$

with periodic boundary conditions over the 'cell', where $\langle$.$\rangle is the averaging operator over the cell (see Appendix$ B) and where the cell can be taken as the whole domain. The effective elastic stiffness tensor $\mathbf{c}^{*}$ is then obtained from the two order 4 intermediate tensors $\mathbf{G}$ and $\mathbf{H}$ with :

$$
\mathbf{c}^{*}=\mathcal{F}_{\lambda_{0}}(\mathbf{H}): \mathcal{F}_{\lambda_{0}}(\mathbf{G})^{-1}
$$

A complete description of deterministic non periodic homogenization for elastic waves can be found in Capdeville 
et al. (2010a) (1D case), Capdeville et al. (2010b) (2D $\mathrm{P}-\mathrm{SV}$ case) and an example of its permitted accuracy is given in Appendix C. The effective tensor is in general fully anisotropic, even for isotropic fine scale model, which is well-known since the work of Backus (1962).

For acoustic media, the same homogenization procedure can be carried out. The involved theoretical development is mathematically the same as the one that can be found in Guillot et al. (2010) for elastic SH waves in 2-D. Applied to the acoustic case, we find that, to compute the effective acoustic wavefield, one has to solve for the effective potential $q^{*}$ and displacement $\mathbf{u}^{*}$ :

$$
\begin{aligned}
& \frac{1}{\kappa^{*}} \ddot{q}^{*}-\nabla \cdot \dot{\mathbf{u}}^{*}=\dot{g} \\
& \dot{\mathbf{u}}^{*}=\mathbf{L}^{*} \cdot \nabla q^{*}
\end{aligned}
$$

where $1 / \kappa^{*}=\mathcal{F}_{\lambda_{0}}(1 / \kappa)$ is once again the filtered version of $1 / \kappa$ and $\mathbf{L}^{*}$ is now an order 2 tensor which may carry the effective anisotropy. This tensor $\mathbf{L}^{*}$ is computed through the acoustic version of the cell problem (as defined in Guillot et al. (2010)), namely by solving over the cell for the vector $\chi$ :

$$
\begin{aligned}
& \nabla \cdot(\mathbf{P})=0 \\
& \mathbf{P}=\frac{1}{\rho} \mathbf{Q} \\
& \mathbf{Q}=\mathbf{I}+\nabla \chi \\
& \langle\chi\rangle=0
\end{aligned}
$$

with periodic boundary conditions over the cell, where $\langle$. is the averaging operator over the cell (see Appendix B), and where once again the cell is usually taken as the whole domain $\boldsymbol{\Omega}$. The effective tensor $\mathbf{L}^{*}$ is then obtained from the two order 2 intermediate tensors $\mathbf{Q}$ and $\mathbf{P}$ with :

$$
\mathbf{L}^{*}=\mathcal{F}_{\lambda_{0}}(\mathbf{P}) \cdot \mathcal{F}_{\lambda_{0}}(\mathbf{Q})^{-1}
$$

In general, $\mathbf{L}^{*}$ is not proportional to the identity, thus leading to an anisotropic effective density. This may appear unusual for the seismology community, however, such an anisotropic effective density has already been encountered for acoustic waves propagating in the so-called sonic crystals (SC), i.e. in fluid with an embedded periodic lattice of cylindrical scatterers (de Hoop, 1995; Cummer and Schurig, 2007; Torrent and Sánchez-Dehesa, 2008), or in layered media with mixed solid and fluids (e.g. Schoenberg, 1984).

At this point, we already see that similar fine scale structures may behave very differently at the effective scale level depending on the type of wave propagation considered (acoustic or elastic). From relations (22-25), it appears that $\mathbf{L}^{*}$ depends only on the spatial distribution of the fine scale density $\rho(\mathbf{x})$ and not on the bulk modulus distribution $\kappa(\mathbf{x})$. On the other hand, from equations (16-18), it appears that the effective elastic stiffness tensor $\mathbf{c}^{*}$ only depends on the fine scale elastic stiffness tensor distribution $\mathbf{c}(\mathbf{x})$ and not on the fine scale density distribution $\rho(\mathbf{x})$. From this observation, one could imagine a medium with a homogeneous fine scale elastic distribution and an heterogeneous density distribution. This would lead to isotropic effective parameters in the elastic case and to large anisotropic effective parameters in the acoustic case. To the other extreme, an heterogeneous fine scale elastic distribution and a homogeneous density distribution would lead to anisotropic effective parameters in the elastic case and to isotropic effective parameters in the acoustic case. Moreover, the fact that the effective 'density tensor' $\mathbf{L}^{*}$ is only an order 2 tensor in 2-D allows only an elliptic anisotropy whereas the effective $\mathbf{c}^{*}$ is an order 4 tensor in 2-D allowing more different types of anisotropy as illustrated on a very simple case in Figures $7 \mathrm{~b}$ and $7 \mathrm{c}$.

As we can see, if the upscaling problems of the elastic and acoustic wave cases are formally very similar they involve different quantities. From the two upscaling problems, there is no reason to expect the same properties from an elastic upscaling and from an acoustic upscaling. Therefore, even if we can build related acoustic and elastic problems at small scale, the respective effective acoustic and elastic media, the ones indeed "seen" by the wavefield, have little chance to be related which explains the numerical observations made in the previous section. To investigate this aspect further, we study in the next section a simple particular medium case.

\section{Isotropic stratified media}

A very useful medium configuration in seismic wave modelling and upscaling is the isotropic stratified medium. This layered medium case reduces to a 1-D problem and displays analytical expressions for computing the effective coefficients as shown by Backus (1962) for the elastic case. Moreover, when layers follow a periodic structure, effective parameters remain constant over the whole domain. This allows to build simple test cases leading to easy interpretation of the results. As an example, we will use the isotropic periodic 2-layer horizontally stratified medium described in Figure 7a. In each layer, density is set using a contrast of $13 \%$ around its average value, and Lamé parameters $\lambda$ and $\mu$ are set using contrasts of $50 \%$ and $68 \%$ around their average value respectively (setting approximatively $\kappa=\lambda+2 \mu$ with a contrast of $68 \%$ around its average value). Each layer is $25 m$ thick, setting the medium's period to $50 \mathrm{~m}$. The source maximum frequency is set such that the minimum wavelength is $\lambda_{P_{m i n}}=400 \mathrm{~m}$, which is indeed much larger than the period $\left(\varepsilon_{0}=0.125\right)$. Snapshots of the energy at $t=5 \mathrm{~s}$ are shown in Figure 7b for the elastic case and in Figure $7 \mathrm{c}$ for the acoustic case, and illustrate the effective anisotropies in each case. In this experiment, three major observations arise :

1. elastic (diamond shaped wave front) effective anisotropy is more complex than acoustic (elliptic) anisotropy;

2. for similar parameters, the acoustic effective aniso- 
tropy is weak compared to the elastic effective anisotropy;

3. effective elastic $\mathrm{P}$-wave and acoustic wave velocities are identical in the vertical direction.

The first point is directly related to the general homogenization procedures for elastic and acoustic waves developed in the previous section : the different dimensions of the effective elastic and acoustic tensors explain the different natures of the effective anisotropies.

In the following, we focus on the specific analytic results from the homogenization for isotropic stratified media which allow to explain in detail the last two observations.

For 2-D elastic periodic stratified media, effective quantities are computed over the periodic cell as (Backus, 1962) :

$$
\begin{aligned}
\mathbf{c}_{1111}^{*} & =\left\langle\lambda+2 \mu-\frac{\lambda^{2}}{\lambda+2 \mu}\right\rangle+\left\langle\frac{1}{\lambda+2 \mu}\right\rangle^{-1}\left\langle\frac{\lambda}{\lambda+2 \mu}\right\rangle^{2}, \\
\mathbf{c}_{2222}^{*} & =\left\langle\frac{1}{\lambda+2 \mu}\right\rangle^{-1} \\
\mathbf{c}_{1212}^{*} & =\left\langle\frac{1}{\mu}\right\rangle^{-1} \\
\mathbf{c}_{1122}^{*} & =\left\langle\frac{1}{\lambda+2 \mu}\right\rangle^{-1}\left\langle\frac{\lambda}{\lambda+2 \mu}\right\rangle \\
\rho^{*} & =\langle\rho\rangle,
\end{aligned}
$$

where $\langle$.$\rangle denotes the averaging operator over the periodic$ cell (see Appendix B).

In 2-D acoustic periodic stratified media, the effective density tensor's analytical expression is computed similarly as for a static scalar problem (Lurie and Cherkaev, 1986; Bosse and Showalter, 1989; Hornung, 1992) and effective parameters are then given by :

$$
\begin{aligned}
\mathbf{L}^{*} & =\left[\begin{array}{cc}
\left\langle\frac{1}{\rho}\right\rangle & 0 \\
0 & \frac{1}{\langle\rho\rangle}
\end{array}\right], \\
\frac{1}{\kappa^{*}} & =\left\langle\frac{1}{\kappa}\right\rangle .
\end{aligned}
$$

Therefore in order to upscale such a medium only 3 simple quantities need to be computed : $\langle\rho\rangle,\langle 1 / \rho\rangle$ and $\langle 1 / \kappa\rangle$.

At this point, the effective kinematic equivalence between elastic $\mathrm{P}$-waves and acoustic waves in the vertical direction (observation 3) can easily be confirmed. Indeed, noticing that for our acoustic parameterization $\kappa=$ $\rho V^{2}=\rho V_{P}^{2}=\lambda+2 \mu$, and using (28), (31), $\mathbf{L}_{22}^{*}$ expression from (32), and (33), we obtain :

$$
V_{P V}{ }^{*}=\sqrt{\frac{\mathbf{c}_{2222}^{*}}{\rho^{*}}}=\sqrt{\kappa^{*} \mathbf{L}_{22}^{*}}=V_{V}{ }^{*}
$$

which means that elastic P-waves and acoustic waves travel vertically with the same effective velocity (respectively $V_{P V}{ }^{*}$ and $\left.V_{V}{ }^{*}\right)$.
To explain the differences in amplitude of effective anisotropies (observation 2), we use a "Thomsen-like" (Thomsen, 1986) measurement of anisotropy, $\epsilon$, in both elastic and acoustic cases. In the elastic case, $\epsilon$ is exactly the Thomsen parameter $\epsilon=\left(\mathbf{c}_{1111}^{*}-\mathbf{c}_{2222}^{*}\right) /\left(2 \mathbf{c}_{2222}^{*}\right)$, which, for a 2-layered medium, can be computed as :

$$
\epsilon=\frac{\nu_{\kappa}^{2}}{2\left(1-\nu_{\kappa}^{2}\right)}\left[1-\left(\frac{\langle\lambda\rangle}{\langle\kappa\rangle} \frac{\nu_{\lambda}}{\nu_{\kappa}}\right)^{2}\right],
$$

where $\kappa=\lambda+2 \mu$ with $\lambda$ and $\mu$ the Lamé parameters, $\langle\lambda\rangle$ and $\langle\kappa\rangle$ denote the average values of $\lambda$ and $\kappa$ respectively, and $\nu_{\lambda}$ and $\nu_{\kappa}$ are their respective contrasts.

In the acoustic case, we similarly use $\epsilon=\left(\mathbf{L}_{11}^{*}-\mathbf{L}_{22}^{*}\right) /\left(2 \mathbf{L}_{22}^{*}\right)$, which, for a 2-layered medium, can be computed as :

$$
\epsilon=\frac{\nu_{\rho}^{2}}{2\left(1-\nu_{\rho}^{2}\right)},
$$

where $\nu_{\rho}$ is the contrast on the density.

First, we note that relations (35) and (36) show some formal similarities as both of them relate effective anisotropy to contrasts on the heterogeneities with the following pattern : the higher the contrasts are $(\nu \longrightarrow 1)$, the more important the effective anisotropy is. In our example, contrast on density was much smaller than contrasts on Lamé parameters and we therefore have $\epsilon \simeq 38.7 \%$ in the elastic case corresponding to a strong anisotropy, while we have $\epsilon \simeq 0.9 \%$ in the acoustic case, corresponding to almost no anisotropy as observed. However, (35) and (36) show directly that the important quantities related to effective anisotropy are fundamentally different between the elastic and the acoustic cases. In the elastic case, effective anisotropy depends only on contrasts on the Lamé parameters while in the acoustic case, effective anisotropy depends only on contrasts on the density. Therefore, if effective anisotropies were already of different kinds (observation 1), relations (35) and (36) show very simply in the periodic 2-layer media case that they are also different in their strength as density's contrasts and Lamé parameters' contrasts are not necessarily correlated, especially for shallow geological structures of the earth. Figure $7 d$ shows the result for an attempt to build a 2-layer acoustic medium at the micoscopic scale with effective kinematic equivalence at least for both radial and transverse directions with the elastic case (i.e. to obtain identical values of $\epsilon$ for the elastic and acoustic cases). In order to obtain such a selective effective kinematic equivalence (acoustic effective anisotropy remains elliptic which still does not account correctly for the diamond shaped elastic effective anisotropy), the construction at the microscopic level of acoustic parameters from the elastic parameters can be easily devised (in a non unique way though, and using effective elastic parameters) for 2-layer periodic media. However, such a mapping between elastic and acoustic parameters is only a mathematical transformation and the physical meaning of the acoustic parameters is here lost. Unfortunately, only a simple kinematic equivalence can 


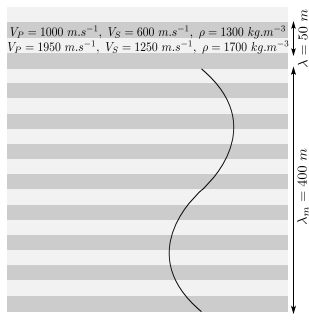

a

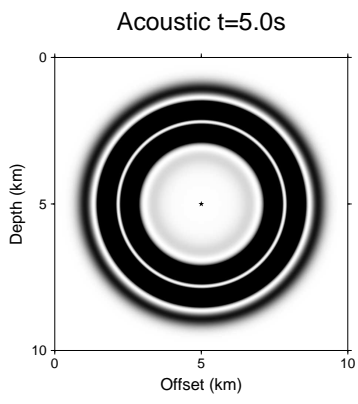

C

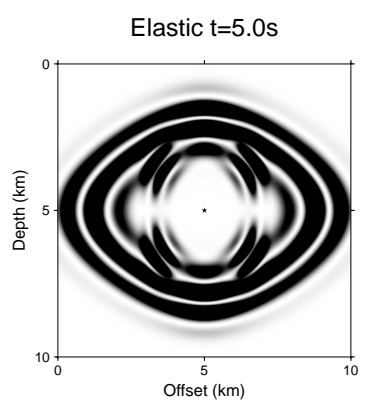

$\mathrm{b}$

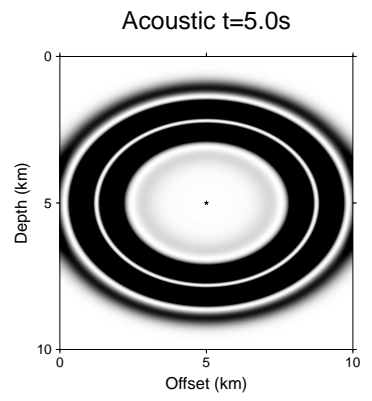

d
Figure 7: Visualisation of effective anisotropy on a 2-layer periodic medium in the elastic and acoustic cases. 7a : Original elastic medium, $7 \mathrm{~b}$ : snapshot of the elastic wavefield, 7c : snapshot of the acoustic wavefield, $7 \mathrm{~d}:$ snapshot of the acoustic wavefield computed in a medium devised from the original elastic medium with the intent to respect effective kinematics in both horizontal and vertical directions.

be achieved following this process. Moreover, this mapping in a two layer case cannot be easily extended to more complex cases, such that we didn't search any further for such kinematic equivalences.

At last, very similar results can be obtained for the continuous case of experiment (1). In particular the vertical effective kinematic equivalence can be seen through the dependence of the error with offset : for low offsets, waves propagate nearly vertically and no delay has to be observed whereas for larger offset full anisotropy has to be considered for phase comparison of the signals. Then, lower contrast on the density compared to contrasts on the elastic moduli implies that acoustic effective anisotropy is weaker than elastic effective anisotropy, which corresponds to the observed delay of the acoustic signal toward the elastic signal.

\section{CONCLUSION}

The acoustic approximation of elastic waves is a common approximation in exploration geophysics. In the present work, we have addressed the validity of this approximation regarding small heterogeneity scales with respect to the wavelength of the wavefield. We have shown that, if the approximation remains valid for isotropic homogeneous media or isotropic heterogeneous media with smooth vari- ations with respect of the wavefield's wavelength, for heterogeneous rough media, i.e. when variations in the elastic parameters are small compared to the wavelengths of the wavefield, this approximation is not reliable anymore. Indeed, forgetting about amplitude errors due to unmodeled P-to-S conversions by acoustic equations, phase errors still arise due to differences in kinematics perceived by the waves between the elastic and acoustic case. Homogenization procedures - for acoustic waves on one hand and for elastic waves on the other hand - offer a way to understand the differences in effective kinematics through the computation of effective parameters and effective anisotropy. Two main reasons arise to explain such effective kinematic differences. First, acoustic effective anisotropy, which is carried by an effective anisotropic density, is systematically reduced to an elliptic anisotropy while elastic effective anisotropy does not suffer such restriction. Second, even if acoustic parameters are related to elastic parameters at the original small scale it is generally not the case anymore for effective parameters because relevant quantities for computing effective parameters (density for the acoustic case, elastic moduli for the elastic case) are not the same. If these two results are available for general heterogeneous media, the particular case of stratified periodic media allowed a more detailed investigation by offering clear relations between parameters of the micro scale medium and effective anisotropy.

Our work shows that if using the acoustic approximation for numerical experiments for full waveform inversion is still possible and pertinent, some care has to be given on what parameters to work on to fully mimic the elastic case. Indeed, it is sometime the $1 / \kappa$ parameter that is chosen to work on and to be inverted for (e.g. Métivier et al. (2013)). Such a choice is equivalent to work on and to invert only for the density in the elastic case, which is possible, but missing a lot of the realistic complexity. Inverting for the density, or better for $\mathbf{L}$, is a better choice, that makes it possible to account for some of the elastic complexity still in the acoustic framework.

In the end, using equations of acoustics as an acoustic approximation for elastic $\mathrm{P}$-waves seemed natural at first but this approximation remains too simple to keep a good accuracy and a physical equivalence in highly heterogeneous - or 'rough' - media.

\section{AKNOWLEDGMENTS}

This work was funded by the ANR blanche "mémé" grant (ANR-10-BLAN-613 MEME). Computations were performed on the CCIPL computer "Erdre". We thank Gaetano Festa for letting us use and modify his 2-D spectral element program and James Rickett fo letting us use and modify the 2-D crosscut of SEG's SEAM model. Last but not least, we want to thank Dr. Fichtner, Dr. Oprsal, and four other anonymous reviewers for their numerous constructive comments, which allowed to improve significantly the quality of this paper. 
APPENDIX A

\section{FROM ELASTIC TO ACOUSTIC EQUATIONS : THE HOMOGENEOUS ISOTROPIC CASE}

The only case for which the elastic and acoustic wave equations can exactly be related is for an infinite isotropic homogeneous domain and for an explosive isotropic source.In such a case, acoustic equations derive from elastic equations as follows. In an isotropic homogeneous domain, the elastic medium is fully described by its density $\rho$ and the two Lamé parameters $\lambda$ and $\mu$, which are constant over the domain. Then, using (4) in (1), we obtain :

$$
\rho \ddot{\mathbf{u}}-\lambda \nabla \cdot[(\nabla \cdot \mathbf{u}) \mathbf{I}]-\mu \nabla \cdot(\nabla \mathbf{u})-\mu \nabla \cdot\left({ }^{T} \nabla \mathbf{u}\right)=\mathbf{f} .
$$

where $\mathbf{f}=\nabla\left(\delta\left(\mathbf{x}-\mathbf{x}_{0}\right)\right.$ Ricker $\left.(t)\right)$ is the explosive source term, with $\mathbf{x}_{0}$ the position of the source, $\delta(\mathbf{x})$ the Dirac distribution, and Ricker $(t)$ the source time function as a Ricker. Using the following identities :

$$
\begin{aligned}
\nabla \cdot[(\nabla \cdot \mathbf{u}) \mathbf{I}] & =\nabla(\nabla \cdot \mathbf{u}) \\
\nabla \cdot\left({ }^{T} \nabla \mathbf{u}\right) & =\nabla(\nabla \cdot \mathbf{u}) \\
\nabla \cdot(\nabla \mathbf{u}) & =\nabla(\nabla \cdot \mathbf{u})-\nabla \times(\nabla \times \mathbf{u})
\end{aligned}
$$

the elastic wave equation can be rewritten as :

$$
\rho \ddot{\mathbf{u}}-\nabla[(\lambda+2 \mu) \nabla \cdot \mathbf{u}]+\mu \nabla \times(\nabla \times \mathbf{u})=\mathbf{f}
$$

where $\nabla \times \mathbf{u}$ denotes the curl operator over vector $\mathbf{u}$.

Assuming that the source is not generating shear waves, only $\mathrm{P}$ waves are propagating in $\boldsymbol{\Omega}$ and, knowing that $P$ waves are irrotational, we have $\nabla \times \mathbf{u}=\mathbf{0}$. In such a case, the displacement vector $\mathbf{u}$, or the velocity vector $\mathbf{v}=\dot{\mathbf{u}}$, derives from a potential $q$. The density being constant, we can choose the potential $q$ such as :

$$
\mathbf{v}=\dot{\mathbf{u}}=\frac{1}{\rho} \nabla q
$$

Differentiating (A-5) over time, using (A-6), assuming the source also derives from a potential $g$ such that :

$$
\mathbf{f}=\nabla((\lambda+2 \mu) g)
$$

which is possible with the explosive source mentioned above, and setting $\kappa=\lambda+2 \mu=\rho V_{P}^{2}$, we obtain :

$$
\nabla\left(\frac{1}{\kappa} \ddot{q}-\nabla \cdot \mathbf{v}-\dot{g}\right)=0
$$

which implies (7). Therefore, in an infinite homogeneous isotropic medium with the acoustic density set to the elastic density and the sound speed set exactly to the elastic P-waves velocity, $V=V_{P}$, and assuming that no shear wave has been generated by the source, the acoustic equations $(7,8)$ have the same solution as the elastic wave equations.
APPENDIX B

\section{FILTERING AND AVERAGING OPERATORS}

We define here the averaging $\langle$.$\rangle and filtering \mathcal{F}_{\lambda_{c}}($.$) oper-$ ators. For a quantity $h(\mathbf{x})$ defined over a $2 D$ cell $\boldsymbol{\Omega}$, the corresponding average is defined as

$$
\langle h\rangle=\frac{1}{|\mathbf{\Omega}|} \int_{\mathbf{\Omega}} h(\mathbf{x}) d \mathbf{x}
$$

where $|\boldsymbol{\Omega}|$ is a measure (e.g. the area) of the cell.

For any function $h$, we define its $2 D$ Fourier transform as

$$
\hat{h}(\mathbf{k})=\int_{\mathbb{R}^{2}} h(\mathbf{x}) \exp (\imath \mathbf{k} \cdot \mathbf{x}) d \mathbf{x}
$$

where $\mathbf{x}$ is the position vector and $\mathbf{k}$ is the wavenumber vector. For any wavenumber vector $\mathbf{k}$ we define its associated wavelength $\lambda_{\mathbf{k}}=1 /|\mathbf{k}|$. To remove any spatial variations smaller than $\lambda_{c}$ of a function $h(\mathbf{x})$ we define the low-pass filter operator $\mathcal{F}_{\lambda_{c}}($.$) as$

$$
\mathcal{F}_{\lambda_{c}}(h)(\mathbf{x})=\int_{\mathbb{R}^{2}} h\left(\mathbf{x}^{\prime}\right) w_{\lambda_{c}}\left(\mathbf{x}-\mathbf{x}^{\prime}\right) d \mathbf{x}^{\prime}
$$

where $w_{\lambda_{c}}$ is a wavelet. Ideally, $w_{\lambda_{c}}$ is such that

$$
\hat{w}_{\lambda_{c}}(\mathbf{k})= \begin{cases}1 & \text { for }|\mathbf{k}| \leq k_{c} \\ 0 & \text { for }|\mathbf{k}|>k_{c}\end{cases}
$$

where $k_{c}=1 / \lambda_{c}$. In practice, we use a wavelet with a finite spatial support. We first define the mother wavelet $w(\mathbf{x})$ such as its power spectrum is

$$
\hat{w}(\mathbf{k})= \begin{cases}1 & \text { for }|\mathbf{k}| \leq a, \\ \frac{1}{2}\left[1+\cos \left(\pi \frac{|\mathbf{k}|-a}{b-a}\right)\right] & \text { for }|\mathbf{k}| \in] a ; b[ \\ 0 & \text { for }|\mathbf{k}| \geq b,\end{cases}
$$

where $a$ and $b$ are two reals around 1 defining the tapper transition between 1 and 0 of the low pass filter. The wavelet in the space domain is then obtained with a Hankel transform

$$
w(\mathbf{x})=\int_{0}^{+\infty} \hat{w}(\mathbf{k}) J_{0}(|\mathbf{k}||\mathbf{x}|)|\mathbf{k}| d|\mathbf{k}|
$$

where $J_{0}$ is the Bessel function of the first kind of order 0 . At last, we define the filter wavelet $w_{\lambda_{c}}(\mathbf{x})$ of corner frequency $k_{c}=1 / \lambda_{c}$ as $w_{\lambda_{c}}(\mathbf{x})=k_{c} w\left(k_{c} \mathbf{x}\right)$.

\section{APPENDIX C \\ NUMERICAL PRECISION AND PHASE SHIFT}

We show here that phase shifts observed between elastic and acoustic pressure signals in the rough cases are not numerical artifacts due to SEM simulations and are related to small scale heterogeneities through the homogenization procedure. To show that there is no precision loss due to spectral element procedure, we use the experiment 2 (random medium) in the rough case configuration with 2 different numerical set-up. The first set-up is defined as 
previously, with $800 \times 900$ square elements of $20 \times 20 \mathrm{~m}^{2}$ with constant elastic properties and with 5 GLL points per space direction. The second set-up is different from the first only by refining the mesh (and time step) by a factor 2 in each direction. Figure $\mathrm{C}$-1a shows on the reflected wave recorded at receiver 1 that the first set-up was already converged and the relative error $\left|\left(p_{1}-p_{2}\right) / p_{2}\right|$ on the whole pressure signal is no more than $1 \%$ for both elastic and acoustic runs.

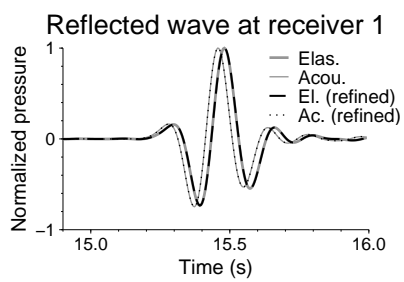

a

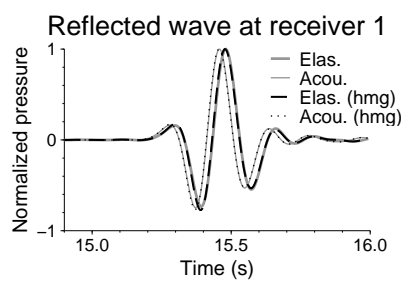

b
Figure C-1: Experiment (2), rough medium : Numerical convergence of the SEM computations (Figure C-1a) and of the homogenization procedure (Figure C-1b) on the reflected wave recorded at receiver 1 .

To numerically show that homogenization theory fully explains the observed phase shifts between elastic and acoustic waveforms we homogenized the random medium of experiment 2 in both elastic and acoustic cases, with an homogenization parameter of $\varepsilon_{0}=0.5$ (which is generally small enough to obtain good accuracy). Then we compute the wavefield in both elastic and acoustic cases using the homogenized media and with the same numerical set-up as in the paper's body (first set-up). We compare then pressure signals computed in the original medium and in the homogenized medium for both the elastic and acoustic cases. Figure $\mathrm{C}-1 \mathrm{~b}$ shows the waveform recorded at receiver 1 in the time window corresponding to the reflected wave. Further convergence analysis of the homogenization procedure can be found in Capdeville et al. (2010b) for the elastic case and Guillot et al. (2010) for the acoustic case.

\section{REFERENCES}

Alkhalifah, T., 1998, Acoustic approximations for processing in transversely isotropic media: Geophysics, 63, $623-631$.

- 2000, An acoustic wave equation for anisotropic media: Geophysics, 65, 1239-1250.

- 2003, An acoustic wave equation for orthorhombic anisotropy: Geophysics, 68, 1169-1172.

Allaire, G., 1992, Homogenization and two-scale convergence: SIAM J. Math. Anal., 23, 1482-1518.

Auriault, J.-L., and E. Sanchez-Palencia, 1977, Étude du comportement macroscopique d'un milieu poreux saturé déformable.: J. Mécanique, 16, 575-603.

Backus, G., 1962, Long-wave elastic anisotropy produced by horizontal layering: Journal of Geophysical Research, 67, 4427-4440.
Bakker, P. M., and E. Duveneck, 2011, Stability analysis for acoustic wave propagation in tilted ti media by finite differences: GJI, 185, 911-921.

Barnes, C., and M. Charara, 2008, Full-waveform inversion results when using acoustic approximation instead of elastic medium: SEG annual meeting, Las Vegas, USA, 1895-1899.

Ben-Hadj-Ali, H., S. Operto, and J. Virieux, 2008, Velocity model building by $3 \mathrm{~d}$ frequency-domain, fullwaveform inversion of wide-aperture seismic data: Geophysics, 73, VE101-VE117.

Bensoussan, A., J.-L. Lions, and G. Papanicolaou, 1978, Asymptotic Analysis of Periodic Structures: North Holland.

Berkhout, A. J., 1984, Seismic migration: Imaging of acoustic energy by wave field extrapolation: Elsevier.

Bosse, M.-p., and R. E. Showalter, 1989, Homogenization of the layered medium equation: Applicable Analysis, 32, 183-202.

Bube, K. P., T. Nemeth, J. P. Stefani, R. Ergas, W. Liu, K. T. Nihei, and L. Zhang, 2012, On the instability in second-order systems for acoustic vti and tti media: Geophysics, 77, T171-T186.

Bunks, C., F. M. Saleck, S. Zaleski, and G. Chavent, 1995, Multiscale seismic waveform inversion: Geophysics, 60, 1457-1473.

Capdeville, Y., L. Guillot, and J.-J. Marigo, 2010a, 1$\mathrm{d}$ non periodic homogenization for the wave equation: GJI, 181, 897-910.

— , 2010b, 2-d non-periodic homogenization to upscale elastic media for p-sv waves: GJI.

Chu, C., B. K. Macy, and P. D. Anno, 2012, Pure acoustic wave propagation in transversely isotropic media by the pseudospectral method: Geophysical Prospecting, 112.

Cummer, S. A., and D. Schurig, 2007, One path to acoustic cloaking: New Journal of Physics, 9, 45.

de Hoop, A., 1995, Handbook of radiation and scattering of waves, 1995.

Etgen, J., S. H. Gray, and Y. Zhang, 2009, An overview of depth imaging in exploration geophysics: Geophysics, 74, WCA5-WCA17.

Fehler, M., and K. Larner, 2008, Seg advanced modeling (seam): Phase i first year update: The Leading Edge, 27, 1006-1007.

Festa, G., and J.-P. Vilotte, 2005, The newmark scheme as velocity-stress time staggering : an efficient implementation for spectral element simulations of elastodynamics: GJI, 161, 789-812.

Fletcher, R., X. Du, and P. J. Fowler, 2008, A new pseudoacoustic wave equation for ti media: SEG annual meeting, Las Vegas, USA, 2082-2086.

Gauthier, O., J. Virieux, and A. Tarantola, 1986, Twodimensional nonlinear inversion of seismic waveforms: Numerical results: Geophysics, 51, 1387-1403.

Grechka, V., L. Zhang, and J. W. R. III, 2004, Shear waves in acoustic anisotropic media: Geophysics, 69, 
$576-582$.

Guillot, L., Y. Capdeville, and J.-J. Marigo, 2010, 2-d non periodic homogenization for the sh wave equation: GJI, 182, 1438-1454.

Hobro, J. W., C. H. Chapman, and J. O. Robertsson, 2014, A method for correcting acoustic finite-difference amplitudes for elastic effects: Geophysics, 79, T243T255.

Hornung, U., 1992, Applications of the homogenization method to flow and transport in porous media: Summer School on Flow and Transport in Porous Media, 167222 .

Komatitsch, D., and J. Tromp, 2002, Spectral-element simulations of global seismic wave propagation, part ii : 3-d models, oceans, rotation and gravity: GJI, 303-318.

Komatitsch, D., and J.-P. Vilotte, 1998, The spectral element method : an effective tool to silmulate the seismic response of $2 \mathrm{~d}$ and $3 \mathrm{~d}$ geological structures: Bulletin of the seismologic Society of America, 368-392.

Lamé, G., 1852, Leons sur la théorie mathématique de l'élasticité des corps solides: Gauthier-Villars.

Landau, L., and E. Lifshitz, 1959a, Fluid mechanics, 1959: Course of Theoretical Physics.

Landau, L. D., and E. M. Lifshitz, 1959b, Course of theoretical physics vol 7: Theory and elasticity: Pergamon Press.

Lurie, K. A., and A. V. Cherkaev, 1986, Exact estimates of the conductivity of a binary mixture of isotropic materials: Proceedings of the Royal Society of Edinburgh: Section A Mathematics, 104, no. 1-2, 21-38.

Métivier, L., R. Brossier, J. Virieux, and S. Operto, 2013, Full waveform inversion and the truncated newton method: SIAM Journal on Scientific Computing, 35, B401-B437.

Operto, S., J. Virieux, A. Ribodetti, and J. E. Anderson, 2009, Finite-difference frequency-domain modeling of viscoacoustic wave propagation in $2 \mathrm{~d}$ tilted transversely isotropic (tti) media: Geophysics, 74, T75-T95.

Plessix, R.-E., G. Baeten, J. W. de Maag, M. Klaassen, Z. Rujie, T. Zhifei, et al., 2010, Application of acoustic full waveform inversion to a low-frequency large-offset land data set: Presented at the 2010 SEG Annual Meeting, Society of Exploration Geophysicists.

Pratt, R. G., C. Shin, and G. Hick, 1998, Gauss-newton and full newton methods in frequency-space seismic waveform inversion: Geophysical Journal International, 133, 341-362.

Sanchez-Palencia, E., 1980, 5, in Non homogeneous media and vibration theory: Springer, Berlin.

Schiemenz, A., and H. Igel, 2013, Accelerated 3-D full-waveform inversion using simultaneously encoded sources in the time domain: application to Valhall ocean-bottom cable data: Geophysical Journal International.

Schoenberg, M., 1984, Wave propagation in alternating solid and fluid layers: Wave motion, 6, 303-320.

Shin, C., and Y. H. Cha, 2008, Waveform inversion in the laplace domain: Geophysical Journal International, 173, 922-931.

Tarantola, A., 1984, Inversion of seismic reflection data in the acoustic approximation: Geophysics, 49, 12591266.

Thomsen, L., 1986, Weak elastic anisotropy: Geophysics, 51, 1954-1966.

Torrent, D., and J. Sánchez-Dehesa, 2008, Anisotropic mass density by two-dimensional acoustic metamaterials: New Journal of Physics, 10.

Vigh, D., B. Starr, J. Kapoor, H. Li, et al., 2010, 3d full waveform inversion on a gulf of mexico waz data set: Presented at the 2010 SEG Annual Meeting, Society of Exploration Geophysicists.

Virieux, J., and S. Operto, 2009, An overview of fullwaveform inversion in exploration geophysics: Geophysics, 74, WCC1-WCC26.

Wu, Z., and T. Alkhalifah, 2014, The optimized expansion based low-rank method for wavefield extrapolation: Geophysics, 79, T51-T60. 